\title{
Differential acquisition of discriminated autoshaping as a function of stimulus qualities and locations
}

\author{
CRAIG A. BOWE \\ Central Institute for the Deaf, St Louis, Missouri \\ LEONARD GREEN \\ Washington University, St. Louis, Missouri \\ and \\ JAMES D. MILLER \\ Central Institute for the Deaf, St. Louis, Missouri
}

\begin{abstract}
In each of two experiments, different groups of pigeons were required to discriminate between one of two basic kinds of stimulus differences: stimulus quality or stimulus location. For stimulusquality groups, a key was illuminated by one of two colors on trials ending with food delivery and by the other color on trials ending with no food. For stimulus-location groups, a key was illuminated at one of two locations on trials ending with food delivery and at the other location on trials ending with no food. The birds began to respond differentially to the stimuli (i.e., peck the keys on food trials and not peck the keys on no-food trials) earlier in acquisiton if the stimulus qualities served as the signals for trial outcomes than if the stimulus locations served as those signals. The results from both experiments are consistent with predictions from a hypothesis regarding interactions among the qualities and locations of stimuli and responses (the "qualitylocation hypothesis"). Furthermore, the present results support other recent demonstrations of the important role that spatial relations among stimuli can play in classical conditioning.
\end{abstract}

Previous research concerning the role of spatial relations in classical conditioning has focused on the necessary and sufficient conditions for the acquisition of signal value by a CS (e.g., Marshall, Gokey, Green, \& Rashotte, 1979; Rescorla \& Cunningham, 1979; Testa, 1975). Those studies asked how the spatial relations between a CS and a US or between a first- and a secondorder CS can affect the development and expression of a conditioned response. Another way in which a spatial relation might be an important factor in classical conditioning, however, is suggested by Szwejkowska's (1967) experiment and by some informal observations made by Bowe, Miller, and Green (1984) during a series of instrumental-conditioning experiments.

Both Szwejkowska's and Bowe et al.'s studies involved an effect of spatial relations on the acquisition of a discrimination between two stimuli, rather than on the acquisition of a conditioned response to a single stimulus. In both cases, differential responding was more readily established if the stimuli differed in "quality" (i.e., sound

Preparation of this paper was supported by Program Project Grant NS 03856 from the National Institute of Neurological and Communicative Disorders and Stroke to Central Institute for the Deaf. The authors thank W. Clark and $\mathbf{J}$. Weisenberger for their comments on the manuscript. Reprint requests may be sent to Leonard Green, Department of Psychology, Washington University, St. Louis, MO 63130. of metronome vs. buzzer in Szwejkowska's experiment and red vs. green signal lights in Bowe et al.'s experiment) than if they differed in "location" (i.e., front vs. back in Szwejkowska's experiment and up vs. down in Bowe et al.'s experiment). For example, in Bowe et al.'s experiment, a red signal light for some pigeons and an upper signal light for others was illuminated on "go" trials during which pecks on a response key produced food. (Their Experiment 1 explicitly involved an instrumental-conditioning procedure, but the implicit contingencies between different signal-light colors and the presence or absence of food at the end of each trial legitimizes the present discussion of the classicalconditioning aspects of the experiment.) A green signal light for the former pigeons and a lower signal light for the latter pigeons was illuminated on "no-go" trials during which neither pecks on the response key nor any other behavior could produce food. The birds for which stimulus qualities (red or green signal lights) were differentially correlated with the presence and absence of food rapidly acquired "sign-tracking" behaviors (Hearst \& Jenkins, 1974) with respect to those stimuli. That is, on many "go" trials, before pecks were directed at the response key, the pigeons approached and pecked the red signal light. They did not approach or contact the green signal light during "no-go" trials. Such behavior with respect to the upper 
and lower signal lights was infrequently observed (informally) in the case of the pigeons for which these stimulus locations were differentially correlated with the presence and absence of food, particularly not in the first few sessions of exposure to the stimuli.

Unfortunately, neither of the two experiments noted above clearly demonstrated a special effect of a spatial relation in a classical-conditioning discrimination. Szwejkowska's conclusions were based largely on her finding that during test trials there was a lack of responding to a stimulus quality that had been paired with the absence of the US, even though the stimulus quality now appeared in a location that had previously been paired with the presence of the US. Such lack of responding, however, may have been more the result of the presentation of an unfamiliar stimulus-quality/stimulus-location combination than the result of any previously conditioned aspect of the stimulus quality. This interpretation gains support from the fact that, during other test trials in her experiment, responding to a stimulus quality that had been paired with the presence of the US was not consistently found when that stimulus quality appeared in a location that had previously been paired with the absence of the US. The observations of Bowe et al. were not only informal, but were also made within the context of instrumental-conditioning discriminations. Thus, the observed behaviors may have been contaminated by various influences of the responsereinforcer contingency.

As a follow-up to the informal observations of Bowe et al., the present experiments were formal tests of the possibility that a stimulus-quality difference would yield more rapid acquisition by pigeons of a simple discriminated autoshaping procedure than would a stimuluslocation difference. Stimulus qualities were defined in terms of two colors, red and green, that could appear on either of two response keys in a standard pigeon chamber. Stimulus locations were defined in terms of the locations of the two response keys-one on the left side and one on the right side of the front panel in the chamber. On half of the trials during each session $(S+$ trials), food was delivered following illumination of one of the response keys. On the other half of the trials (S- trials), no food was delivered following response-key illuminations. Some birds were exposed to stimulus-quality (SQ) discriminations in which one color (e.g., red) signaled $S+$ trials ending with food delivery and the other color (e.g., green) signaled S- trials ending with no food. Other birds were exposed to stimulus-location (SL) discriminations in which illuminations of one location (e.g., left response key) signaled $S+$ trials ending with food delivery and illuminations of the other location (e.g., right response key) signaled $\mathbf{S}-$ trials ending with no food. The number of "correct" trials (i.e., trials in which at least one peck occurred during $S+$ and trials in which no pecks occurred during $\mathbf{S}-$ ) per session served as the dependent variable in the present experiments.

\section{EXPERIMENT 1}

\section{Method}

Subjects

Each of 24 experimentally naive female White Carneaux pigeons was reduced to $80 \%$ of its free-feeding weight. The birds were maintained at that weight with supplementary feedings after experimental sessions. Water and grit were freely available in each bird's home cage.

\section{Apparatus}

A Gerbrands pigeon chamber measuring $30 \mathrm{~cm}$ across the front panel, $32 \mathrm{~cm}$ front to back, and $30 \mathrm{~cm}$ high was used. Two horizontally aligned response keys, mounted behind circular holes $(2-\mathrm{cm}-$ diam) in the front panel, could be transilluminated with either red or green light. The colors were produced by supplying $28 \mathrm{~V} \mathrm{dc}$ to Type 1829 bulbs that were covered with red or green plastic caps. The centers of the keys were $7.5 \mathrm{~cm}$ below the ceiling, $15 \mathrm{~cm}$ apart, and $7.5 \mathrm{~cm}$ from the nearest side wall. A circular (5-cm-diam) opening was centered on the front panel with its lowest point $7 \mathrm{~cm}$ above the floor. A food hopper could be raised behind this opening. When raised, the hopper was illuminated by an 1829 white bulb located in the opening above the hopper and all other sources of illumination in the chamber were extinguished.

The chamber was enclosed in a sound-attenuating box with a ventilating fan running continuously. A houselight (two 1829 bulbs supplied with $28 \mathrm{~V}$ dc and covered by white plastic caps) that was centered on the ceiling of the box $26 \mathrm{~cm}$ from the front panel provided general illumination of the chamber throughout experimental sessions, except when the hopper was raised. The scheduling and recording equipment was located in an adjacent room.

\section{Procedure}

Magazine training. All birds were first exposed to several sessions of magazine training. During each of these sessions, the food hopper was raised on each of $\mathbf{4 0}$ occasions according to an irregular schedule with a mean interval of $45 \mathrm{sec}$ and a range of 8-160 sec. Most birds were eating reliably after two or three of these sessions. Two birds required a fourth session of magazine training.

Discriminated autoshaping. For all birds, during each of the eight daily sessions there were $20 \mathrm{~S}+$ trials (ending with 4-sec food deliveries and a return to the intertrial-interval conditions) and 20 $\mathrm{S}-$ trials (ending with return to the intertrial-interval conditions). During intertrial intervals, the houselight was the only illumination in the chamber. A trial consisted of an 8-sec red or green illumination of one of the two response keys (the other key remained dark), followed by one of the two possible outcomes. Trials occurred according to an irregular schedule with a mean interval of $45 \mathrm{sec}$ and a range of 8-160 sec. Trials were ordered quasirandomly such that no more than three $S+$ or three $S-$ trials could occur in succession. There were two such orderings of trials possible for each group, and which of the two orderings was used for a daily session was randomly determined.

The birds were assigned to three groups of 8 birds apiece: SQ, SL, and SL(C). For the SQ (stimulus quality) group, red or green illuminations of a response key served as the differential signals for food (S+) and no food ( $\mathrm{S}-$ ) independently of whether the colors appeared on the left or the right response key. For half of the birds, red illuminations of either response key were the $S+$ and green illuminations of either response key were the $S-$. For the other half of the birds in the SQ group, green was the S+ and red was the $S-$. Red and green each appeared 10 times on the left response key and 10 times on the right response key for these birds, but the location of the color was uncorrelated with the trial outcome. 
For the SL (stimulus location) group, illuminations of the lefi or right response key served as the differential signals for food and no food independently of whether the key was red or green. For half of the birds, either red or green illumination of the left response key was the $\mathrm{S}+$ and either red or green illumination of the right response key was the S- . For the other half of the birds in the SL group, right was the $S+$ and left was the $S-$. Red and green each appeared 10 times on the left response key and 10 times on the right response key, but the color of the response key was uncorrelated with the trial outcome.

For the SL(C) group, only one color (red or green) was used in the experiment, but, as for the SL group, illuminations of the left or right response key served as the differential signal for food and no food. For half of the birds, illuminations of the left response key were the $\mathrm{S}+$ and illuminations of the right response key were the $S-$. For the other half of the birds, right was the $S+$ and left was the S-. For half of the birds within each subgroup, the keys were always illuminated with red light; for the other half of these birds, the keys were always illuminated with green light.

\section{Results}

Acquisition of discriminated autoshaping is shown in Figure 1 for each of the three groups. The mean number of "correct" trials is shown in Figure 1 for each group as a function of sessions. A "correct" trial was defined as at least one peck on the illuminated key during $S+$ trials or no pecks on the illuminated key during $S$ - trials. Thus, for a given session, 40 correct trials represent perfect discrimination and 20 correct trials represent chance performance.

Inspection of Figure 1 suggests that: (1) all groups eventually acquired their respective discriminations and reached similar, high levels of asymptotic performance, and (2) the SQ group learned fastest and the SL group learned slowest. Statistical analysis of the data supported these observations. A mixed, two-way analysis of vari-

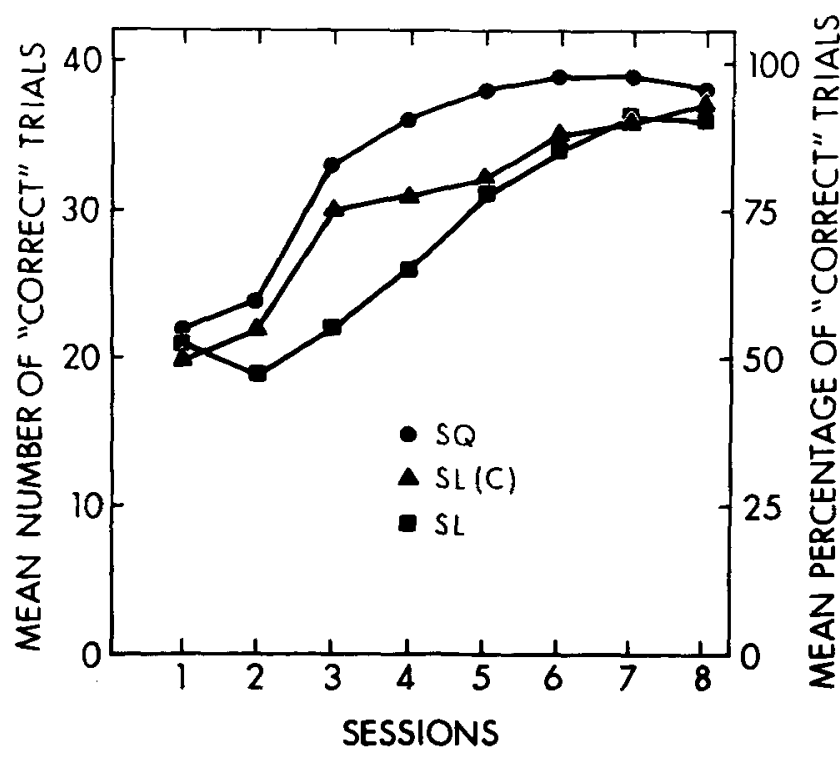

Figure 1. The mean number (left ordinate) and mean percentage (right ordinate) of "correct" trials for each group as a function of sessions in Experiment 1. (See text for definition of "correct.") ance yielded a significant main effect of groups $[F(2,21)$ $=9.20, p<.01]$, a significant main effect of sessions $[F(7,147)=132.5, p<.01]$, and a significant interaction of groups $\times$ sessions $[F(14,147)=3.75, p<.01]$. The sessions effect, of course, simply reflects the learning by each group of its respective discrimination. The groups effect largely reflects the overall superiority of learning and performance on the discriminated autoshaping task shown by the SQ group relative to the SL group, but also partially reflects the differences in learning and performance among all the groups. And, finally, the groups $\times$ sessions interaction reflects the differential learning rates shown by the three groups. However, a post hoc comparison of the overall means for the three groups (Tukey's $a$ test) showed that although the SQ group differed significantly $(p<.05)$ from the SL group, the SL(C) group did not differ from either of the other groups.

The data shown in Figure 2 are the mean number of $S+$ and $S$ - trials with the occurrence of a peck for each group across sessions. These data simply provide for all groups a more detailed picture of the acquisition of keypecking behavior and of the appropriate discriminative performance with respect to $S+$ and $S-$. All groups exhibit a similar overall pattern whereby pecking occurs during both $\mathrm{S}+$ and $\mathrm{S}-$ in the early sessions and decreases during $\mathrm{S}-$ in the final sessions. The groups differ with respect to the session(s) at which the divergence between pecking during $S+$ and during $S$ - occurs, as well as with respect to the rate of decrease in pecking during $S$-. These differences, of course, are the ones reflected in our primary measure of discriminative performance discussed above (see Figure 1).

\section{Discussion}

The present results, as well as those of Bowe et al. (1984) and Szwejkowska (1967), can be interpreted with reference to a hypothesis that was developed to explain differential acquisition rates for instrumental-conditioning discriminations that involve interactions among the qualities and locations of stimuli and responses, the "qualitylocation hypothesis"' (Miller \& Bowe, 1982):

Stimulus qualities are easily associated with (go with) response qualities and stimulus locations are easily associated with (go with) response locations, but stimulus qualities are not easily associated with (do not go with) response locations and stimulus locations are not easily associated with (do not go with) response qualities. (p. 131)

According to Miller and Bowe, an example of a "response-quality" task is the so-called "go/no-go" task in which the subject must respond in the presence of the "go" stimulus to produce reinforcement; in the presence of the "no-go" stimulus, the subject cannot produce reinforcement. An example of a "response-location" task is the so-called "go-left/go-right" task in which, to produce reinforcement, the subject must respond to its left in the presence of the "go-left" stimulus and to its right in the presence of the "go-right" stimulus. The discriminative 


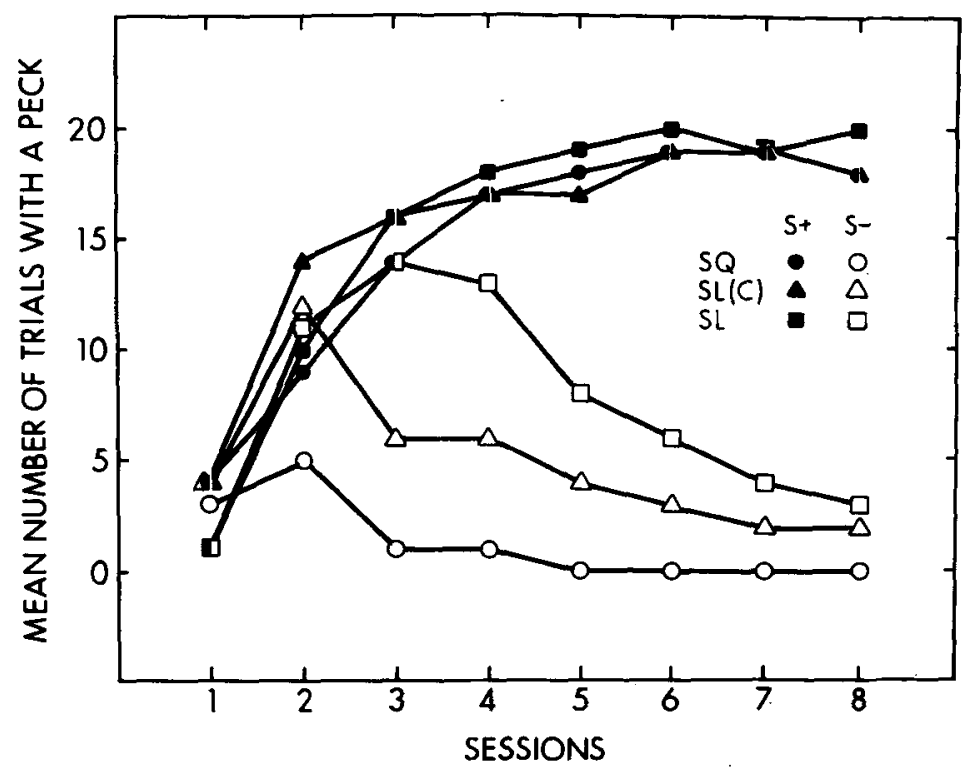

Figure 2. The mean number of $S+$ and $S-$ trials for each group on which a peck occurred as a function of sessions in Experiment 1. Filled symbols represent pecks on $S+$ trials and open symbols represent pecks on $S$ - trials. Circles represent the SQ group, squares represent the SL group, and triangles represent the SL(C) group.

stimuli for response-quality tasks can differ in either stimulus quality (a stimulus-quality/response-quality discrimination) or stimulus location (a stimuluslocation/response-quality discrimination). Similarly, the discriminative stimuli for response-location tasks can differ in either stimulus quality (a stimulusquality/response-location discrimination) or stimulus location (a stimulus-location/response-location discrimination).

If one takes discriminated autoshaping to be a responsequality task due to the go/no-go nature of responding in such procedures, then the present results are consistent with a prediction from the quality-location hypothesis that a stimulus-quality/response-quality discrimination (i.e., the SQ group) should be learned faster than a stimuluslocation/response-quality discrimination [i.e., the SL and SL(C) groups]. Recall, however, that the quality-location hypothesis was formulated within the context of instrumental-conditioning procedures in which trial outcomes are response-dependent, unlike classicalconditioning procedures in which trial outcomes are response-independent. Thus, the present study simply suggests that the quality-location hypothesis may have more general applicability than has been previously indicated (e.g., by Lawicka's, 1964, 1969, data).

Two comments about the performance of the SL(C) group relative to that of the other groups are necessary here. First, the fact that the SQ and SL(C) groups did not significantly differ in favor of more rapid learning by the SQ group weakens somewhat the interpretation offered by the quality-location hypothesis. This is true despite the finding of the significant groups effect, the significant difference between the SQ and SL groups, and the significant groups $\times$ sessions interaction, all of which are predicted by the quality-location hypothesis. Second, although the SL(C) group did not differ significantly in performance from either of the others, its performance was generally between that of the SQ group and the SL group. The SL(C) group was included as a control for the possibility that the random presentations of both colors (red and green) to the SL group might somehow interfere with the birds' ability to respond appropriately to the relevant stimulus locations. Such interference might be expected on the basis of the quality-location hypothesis because stimulus qualities, but not stimulus locations, are supposedly primed for associating under a responsequality task. The finding that the SL(C) group, in which only one color was presented to a given bird, performed slightly (but not significantly) better than did the SL group lends credence to such an interference interpretation. Stated more explicitly, the interference interpretation is that if, during acquisition of a stimulus-location/responsequality discrimination, there is present an irrelevant or "incidental" (see Wagner, 1969) variation in a stimulusquality dimension, then the stimulus-quality variations will interfere with acquisition of the discrimination.

There is an uninteresting but viable alternative explanation for the results obtained in Experiment 1 that does not refer to the quality-location hypothesis. Briefly stated, this alternative contends that because pigeons do not peck (and thus, perhaps, do not readily attend to) dark keys, the birds in the SL and SL(C) groups were at a disadvantage relative to those in the SQ group. That is, one could argue that the location of the illuminated key for the birds 
in the SL and SL(C) groups was functionally defined with respect to the location of the dark key. Because the dark key might not have been "noticed" by any of the birds (at least not during the initial sessions of Experiment 1), birds in the SL and SL(C) groups were slower to learn than were those in the SQ group for which the location of keys, dark or illuminated, was irrelevant with respect to the occurrence of food. Experiment 2 was conducted to test this alternative against the explanation provided by the quality-location hypothesis.

\section{EXPERIMENT 2}

If the basic result from Experiment 1 was due to the difficulty that the birds in the SL group had in attending to the location of the dark key rather than to a kind of quality-location effect, then illumination of both keys for the SQ- and SL-group birds exposed to otherwise identical procedures as were those in the corresponding groups in Experiment 1 should eliminate any performance differences between such groups. Accordingly, in Experiment 2, pigeons were exposed to either a SQ or a SL procedure that was identical to that employed for the birds in Experiment 1, except that the key which would have been dark during trials in Experiment 1 was always illuminated with white light during trials in Experiment 2.

\section{Method}

\section{Subjects and Apparatus}

Sixteen experimentally naive female White Carneaux pigeons were treated and maintained in a manner identical to that employed in Experiment 1. The apparatus was identical to that used in Experiment 1.

\section{Procedure}

Magazine training. All birds were magazine trained in the same fashion as those of Experiment 1. However, most of the birds required 4-5 sessions to begin eating reliably, rather than the 2-3 sessions required by the birds in Experiment 1 .

Discriminated autoshaping. The birds were assigned to two groups of 8 birds apiece: SQ(2) and SL(2). The procedure for the SQ(2) group was identical to that for the SQ group in Experiment 1, except that on any given trial the key that was not illuminated red or green was illuminated white. For half of the birds, red signaled
$S+$ trials and green signaled $S-$ trials. The reverse was true of red and green for the other half of the birds. The procedure for the SL(2) group was identical to that for the SL group in Experiment 1, except that, again, on any given trial the key that was not illuminated red or green was illuminated white. For half of the birds, red or green illuminations of the left key signaled $S+$ trials and red or green illuminations of the right key signaled $S-$ trials. The reverse was true of left and right illuminations for the other half of the birds. All birds were studied daily for 16 sessions.

\section{Results and Discussion}

Acquisition of discriminated autoshaping is shown in Figure 3 for both of the groups, SQ(2) and SL(2). The data from Experiment 2 are displayed in Figure 3 in nearly the same way the data from Experiment 1 were displayed in Figure 1. However, unlike Experiment 1, in which during each trial one of the keys was dark and inoperable, pecks on either key during a trial were included to determine "correct" trials in Experiment 2. Inspection of Figure 3 suggests that: (1) both groups eventually acquired their respective discriminations and reached similar levels of asymptotic performance, and (2) the SQ(2) group learned faster than the SL(2) group. Statistical analysis via a mixed, two-way analysis of variance supported these observations by yielding a significant main effect of groups $[F(1,14)=21.05, p<.01]$, a significant main effect of sessions $[F(15,210)=29.53, p<.01]$, and a significant interaction of groups $\times$ sessions $[F(15,210)$ $=2.11, p<.01]$. Again, the sessions effect reflects the learning by each group of its respective discrimination. The groups effect reflects the overall superiority in learning and performance of the $S Q(2)$ group relative to that of the SL(2) group. And, perhaps most importantly, the groups $\times$ sessions interaction reflects the fact that the SQ(2) group acquired its discrimination faster than the SL(2) group acquired its discrimination.

The data shown in Figure 4 are the mean number of $S+$ and S- trials with the occurrence of a peck for each group across sessions. As was true of the data shown in Figure 2 for Experiment 1, the data shown in Figure 4 for Experiment 2 provide for both groups a more detailed picture of the acquisition of keypecking behavior and of the appropriate discriminative performance with respect

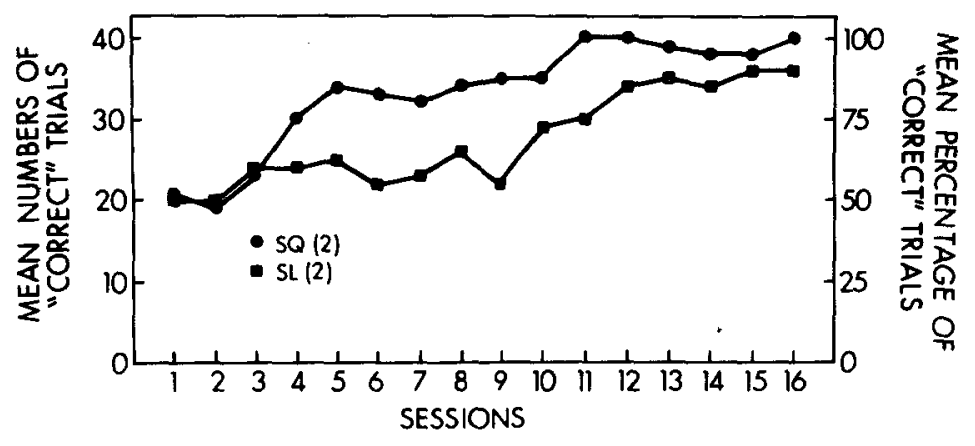

Figure 3. The mean number (left ordinate) and mean percentage (right ordinate) of "correct" trials for each group as a function of sessions in Experiment 2. (See text for definition of "correct.") 


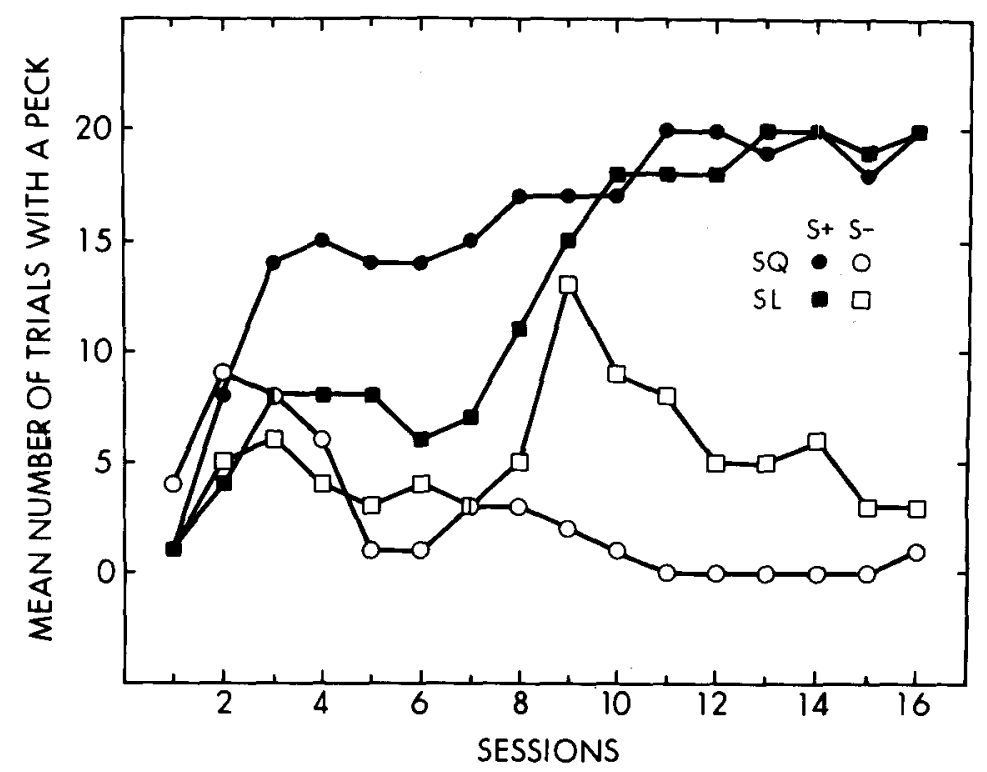

Figure 4. The mean number of $S+$ and $S-$ trials for each group on which a peck occurred as a function of sessions in Experiment 2. Filled symbols represent pecks on $S+$ trials and open symbols represent pecks on $S$ - trials. Circles represent the SQ(2) group and squares represent the SL(2) group.

to $S+$ and $S-$. Both groups begin to peck the keys during $S+$ and $S-$ trials of the first few sessions. However, unlike the SL(2) group, by the fifth session the SQ(2) group had substantially increased the number of $S+$ trials with the occurrence of a peck but had decreased the number of $S$ - trials with the occurrence of a peck to an asymptotic level near zero. It was not until the eighth session that the SL(2) group began to peck the keys on more than half of the S+ trials. Pecking during S- trials in the SL(2) group rose to a peak of about one half of the trials on the ninth session and then decreased to an asymptotic level of about one quarter of the trials. The pattern of results shown in Figure 4 is reflected in our primary measure of discriminative performance discussed above (see Figure 3).

The mean number of $S+$ and $S-$ trials with the occurrence of a peck on the colored and/or white key for each group across sessions is presented in Table 1. The most interesting aspect of these data is that on $\mathrm{S}+$ trials the $S Q(2)$ group quickly came to peck the colored key almost exclusively, whereas the SL(2) group pecked the white key consistently more often than it did the colored key.

It is clear from analyses like Wagner's (1969) why the SQ(2) group learned to peck only the colored keys: The

Table 1

Mean Number of S + and S - Trials with the Occurrence of a Peck on the Colored and/or White Key for Each Group Across Sessions

\begin{tabular}{|c|c|c|c|c|c|c|c|c|}
\hline \multirow[b]{3}{*}{ Session } & \multicolumn{4}{|c|}{ SQ(2): Stimulus Quality } & \multicolumn{4}{|c|}{ SL(2): Stimulus Locations } \\
\hline & \multicolumn{2}{|c|}{$S+$ Trials } & \multicolumn{2}{|c|}{ S- Trials } & \multicolumn{2}{|c|}{$S+$ Trials } & \multicolumn{2}{|c|}{$S-$ Trials } \\
\hline & $\begin{array}{c}\text { Colored } \\
\text { Key }\end{array}$ & $\begin{array}{l}\text { White } \\
\text { Key }\end{array}$ & $\begin{array}{c}\text { Colored } \\
\text { Key }\end{array}$ & $\begin{array}{c}\text { White } \\
\text { Key }\end{array}$ & $\begin{array}{c}\text { Colored } \\
\text { Key }\end{array}$ & $\begin{array}{c}\text { White } \\
\text { Key }\end{array}$ & $\begin{array}{c}\text { Colored } \\
\text { Key }\end{array}$ & $\begin{array}{l}\text { White } \\
\text { Key }\end{array}$ \\
\hline 1 & 4 & 2 & 1 & 3 & 1 & 2 & 1 & 1 \\
\hline 2 & 7 & 4 & 2 & 8 & 1 & 3 & 2 & 3 \\
\hline 3 & 12 & 2 & 2 & 7 & 2 & 6 & 3 & 3 \\
\hline 4 & 15 & 0 & 2 & 5 & 2 & 6 & 2 & 3 \\
\hline 5 & 14 & 0 & 1 & 1 & 1 & 7 & 2 & 2 \\
\hline 6 & 14 & $\mathbf{0}$ & 0 & 0 & $\mathbf{0}$ & 6 & 2 & 2 \\
\hline 7 & 15 & 0 & 0 & 2 & 0 & 6 & 2 & 1 \\
\hline 8 & 17 & $\mathbf{0}$ & 1 & 2 & 1 & 10 & 2 & 3 \\
\hline 9 & 17 & 0 & 1 & 1 & 2 & 13 & 6 & 8 \\
\hline 10 & 16 & 1 & 0 & 1 & 3 & 15 & 5 & 6 \\
\hline 11 & 20 & 1 & 0 & 0 & 4 & 15 & 4 & 6 \\
\hline 12 & 20 & 0 & 0 & 0 & 4 & 17 & 3 & 4 \\
\hline 13 & 19 & 1 & 0 & 0 & 5 & 18 & 3 & 2 \\
\hline 14 & 20 & 0 & 0 & 0 & 3 & 18 & 4 & 2 \\
\hline 15 & 18 & 0 & 0 & 0 & 3 & 16 & 1 & 2 \\
\hline 16 & 20 & 0 & 0 & 1 & 3 & 17 & 1 & 2 \\
\hline
\end{tabular}


$\mathrm{S}+$ color was perfectly correlated with the occurrence of food, whereas the white key was correlated with food on only one half of the trials during a session. (Of course, the S- color was perfectly correlated with the nonoccurrence of food as a trial outcome, but this is not important in the present context of explaining the behavioral differences with respect to colored and white keys.) An explanation for the behavior of the SL(2) group with respect to pecking the white and colored keys is not as straightforward as that for the SQ(2) group. This is because red, green, and white keys were correlated equally often with the occurrence of food for the SL(2) group: Food occurred on only one half of the trials on which each of these kinds of key illumination occurred. Thus, as a pigeon in the SL(2) group learned the required discrimination, it could choose to attend to and peck at the location of the colored key, the location of the white key, or both as the relevant signal for food. Even assuming that the pigeon might reduce attentional demands by choosing one of the first two of these alternatives, we must still explain the birds' observed preference for the white key, given the fact that its correlation with food occurrence was equal to that of the colored key. The fact that these birds pecked most often at the location of the white key suggests a parallel between their behavior and that of pigeons performing a "feature-negative" discrimination (see Hearst's [1978] review of the feature-positive and feature-negative effects and their associated phenomena). In both cases, the birds that eventually learn the discrimination direct much of their pecking at the common element present on the key during both $\mathrm{S}+$ and $\mathrm{S}-$ trials. Perhaps, whatever mechanisms are responsible for the tendency of pigeons to peck at the common element in feature-negative discriminations also operated in Experiment 2 to direct pecking at the white key for the SL(2) birds.

A final observation is that the birds in the SQ(2) and SL(2) groups of Experiment 2 appeared to learn more slowly than their counterparts in the SQ and SL groups of Experiment 1. This slower learning in Experiment 2 may have been due to either one or both of two factors. First, for reasons unknown, the birds in Experiment 2 were more reluctant to eat from the food hopper during magazine training than were those in Experiment 1. Such reluctance obviously could have had fairly pronounced effects on the early acquisition of the discriminations. Second, and perhaps more importantly, the illumination of the white key on every trial in Experiment 2 probably interfered with the learning and/or performance of the discriminations by the birds in Experiment 2 relative to those in Experiment 1, which were not exposed to such illuminations. Despite this potential "interference" effect on the two groups in Experiment 2, and regardless of the precise mechanism by which it might have affected the learning and performance of the two groups, the main conclusion to be reached on the basis of the present results is that a kind of quality-location effect was shown in Experiment 2 by the more rapid acquisition of discriminated autoshaping on the part of the group presented with stimulus qualities, $S Q(2)$, relative to that of the group presented with stimulus locations, SL(2).

\section{GENERAL DISCUSSION}

Pavlov (1927) claimed that "the differentiation of sounds by their direction presents no more difficulty than any other differentiation, and is capable of great precision"' (p. 151). However, the data from the present experiments suggest that the location of conditioned stimuli may affect behavior differently from other kinds of conditioned stimuli in Pavlovian "differentiation," at least in discriminated autoshaping of the pigeon's keypeck by means of visual stimuli. Specifically, we have shown that the learning of a discriminated-autoshaping task proceeds more rapidly if stimulus qualities such as red or green illuminations of a response key are employed as $S+$ and $S$ - than if stimulus locations such as illuminations of a left or a right response key are employed as $\mathbf{S}+$ and $\mathrm{S}-$. In so doing, we have provided confirmation of the informal observations made by Bowe et al. (1984) concerning the differential sign-tracking behaviors that were performed by their birds with respect to stimuli from instrumental-conditioning discriminations. Furthermore, our results support Szwejkowska's (1967) finding that, in classical conditioning, stimulus quality acquires control of discriminated responding more readily than does stimulus location.

There are a number of alternative explanations for the present results. Foremost among them is an explanation in terms of stimulus salience. That is, it may have been that the stimulus-quality differences, red versus green, were more salient than the stimulus-location differences, left versus right. Thus, the pigeons in the SQ and $S Q(2)$ groups learned their discriminations more rapidly than did those in the SL, SL(C), and SL(2) groups. In the instrumental-conditioning experiments that have been cited as the strongest support for the quality-location hypothesis (e.g., Bowe, Miller, \& Green, 1987; Lawicka, 1964, 1969), such a salience alternative has been ruled out via the use of the "double-dissociation" design (Schwartz, 1974). Stimulus qualities were shown to be more effective discriminative stimuli than stimulus locations if the discrimination involved response qualities, but stimulus locations were shown to be more effective discriminative stimuli than stimulus qualities if the discrimination involved response locations. While such a design would be difficult to implement in a classical-conditioning procedure, a classical-conditioning analogue to the responselocation task of an instrumental-conditioning discrimination is possible via preparations other than autoshaping. For example, one could apply localized USs to left and right body parts, as in conditioned eyeblink experiments ${ }^{1}$ (e.g., Wagner, 1969) or conditioned salivary experiments and compare discrimination learning given CS-quality differences with that given CS- location differences. 
Quite apart from their relevance to the quality-location hypothesis, the present results emphasize the importance of experimental investigations into the effects of spatial relations among stimuli on classical conditioning in particular, and on learning and behavior in general (see Bowe, 1984). Although little research in classical conditioning has been concerned with the roles of various spatial relations, there have been some recent efforts to conduct such investigation (e.g., Marshall et al., 1979; Rescorla \& Cunningham, 1979; Testa, 1975). The present experiments join with these recent efforts to encourage further explorations of spatial relations in classical conditioning.

\section{REFERENCES}

Bowe, C. A. (1984). Spatial relations in animal learning and behavior. Psychological Record, 34, 181-209.

Bowe, C. A., Miller, J. D., \& Green, L. (1984). Qualities and locations of stimuli and responses affecting discrimination learning of pigeons. Unpublished manuscript.

Bowe, C. A., Miller, J. D., \& Green, L. (1987). Qualities and locations of stimuli and responses affecting discrimination learning of chinchillas (Chinchilla laniger) and pigeons (Columba livia). Journal of Comparative Psychology, 101, 132-138.

HeARST, E. (1978). Stimulus relationships and feature selection in learning and behavior. In S. Hulse, H. Fowler, \& W. K. Honig (Eds.), Cognitive processes in animal behavior (pp. 51-88). Hillsdale, NJ: Erlbaum.

Hearst, E., \& Jenkins, H. M. (1974). Sign-tracking: The stimulusreinforcer relation in directed action. Austin, TX: Psychonomic Society.

LAWICKA, W. (1964). The role of stimulus modality in successive discrimination and differentiation learning. Bulletin of the Academy of Polish Sciences, 13, 35-38.

LAWICKA, W. (1969). Differing effectiveness of auditory quality and location cues in two forms of differentiation learning. Acta Biologiae Experimentalis, 29, 83-92

Marshall, B. S., Gokey, D. S., Green, P. L., \& Rashotte, M. E. (1979). Spatial location of first-and second-order visual conditioned stimuli in second-order conditioning of the pigeon's keypeck. Bulletin of the Psychonomic Society, 13, 133-136.

Miller, J. D., \& Bowe, C. A. (1982). Roles of the qualities and locations of stimuli and responses in simple associative learning: The quality-location hypothesis. Pavlovian Journal of Biological Science, 17, 129-139.

Pavlov, I. P. (1927). Conditioned reflexes. Oxford: Oxford University Press.

Rescorla, R. A., \& Cunningham, C. L. (1979). Spatial contiguity facilitates Pavlovian second-order conditioning. Joumal of Experimental Psychology: Animal Behavior Processes, 5, 152-161.

SchwarTZ, B. (1974). On going back to nature: A review of Seligman \& Hager's Biological boundaries of learning. Journal of the Experimental Analysis of Behavior, 21, 183-198.

SZWEJKowsKA, G. (1967). Qualitative versus directional cues in differential conditioning: 111 . The role of qualitative and directional cues in differentiation of salivary conditioned reflexes. Acta Biologiae Experimentalis, 27, 413-420.

TESTA, T. J. (1975). Effects of similarity of location and temporal intensity pattern of conditioned and unconditioned stimuli on the acquisition of conditioned suppression in rats. Journal of Experimental Psychology: Animal Behavior Processes, 1, 114-121.

WAGNER, A. R. (1969). Incidental stimuli and discrimination leaming. In R. M. Gilbert \& N. S. Sutherland (Eds.), Animal discrimination learning (pp. 83-111). London: Academic Press.

\section{NOTE}

1. One of the reviewers of the paper pointed out that such lateralized salivary conditioning experiments have been conducted by $K$. S. Abuladze. The monographs have not been translated from the Russian, and so we are unable to evaluate the research.

(Manuscript received May 18, 1984; revision accepted for publication January 16, 1987.) 